Journal of Theoretical and Applied Mechanics, Sofia, Vol. 47 No. 3 (2017) pp. 81-95

\title{
ANALYTICAL SOLUTION OF DISPLACEMENTS AROUND CIRCULAR OPENINGS IN GENERALIZED HOEK-BROWN ROCKS
}

\author{
HOUXU HUANG ${ }^{1 *}$, JiE Li ${ }^{1}$, JiUQi WeI ${ }^{2}$ \\ ${ }^{1}$ State Key Laboratory of Disaster Prevention and Mitigation of Explosion \\ and Impact, PLA University of Science and Technology, Nanjing, China \\ ${ }^{2} 61489$ Army Unit, Luoyang, China
}

[Received 22 September 2016. Accepted 26 June 2017]

\begin{abstract}
The rock in plastic region is divided into numbers of elements by the slip lines, resulted from shear localization. During the deformation process, the elements will slip along the slip lines and the displacement field is discontinuous. Slip lines around circular opening in isotropic rock, subjected to hydrostatic stress are described by the logarithmic spirals. Deformation of the plastic region is mainly attributed to the slippage. Relationship between the shear stresses and slippage on slip lines is presented, based on the study of Revuzhenko and Shemyakin. Relations between slippage and rock failure are described, based on the elastic-brittle-plastic model. An analytical solution is presented for the plane strain analysis of displacements around circular openings in the Generalized Hoek-Brown rock. With properly choosing of slippage parameters, results obtained by using the proposed solution agree well with those presented in published sources.
\end{abstract}

KEY WORDS: Analytical solution, generalized Hoek-Brown yield criterion, slip lines in plastic region, slippage parameter.

\section{INTRODUCTION}

Exact solutions for preliminary design of tunnels, mine shafts and boreholes, are generally required for the validation of numerical methods and computer software. However, compared with exact solutions, the simple closed-form solutions are more preferred in practical engineering. For many types of rocks, the most commonly used liner Mohr-Coulomb (M-C) yield criterion may not be valid, whereas, the nonliner Hoek-Brown (H-B) yield criterion has been proved to be more suitable for description of rock failure [1,2].

Until now, there are many studies about the circular openings in elastic-brittleplastic rock by using the original H-B yield criterion [1,3-6]. However, due to the fact that most of the equations, which are obtained by using the GHB (Generalized

${ }^{*}$ Corresponding author e-mail: wuhanhp14315@163.com 
Hoek-Brown) yield criterion can't be solved with the general methods, thus, the studies about the rock mass governed by the GHB yield criterion are rare. Sofianos and Nomikos proposed an approximate solution for the stresses around the circular openings $[7,8]$. Carranza-Torres agreed with the methods and solutions proposed by Sofianos and Nomikos, and presented his own analytical solutions for the displacements and stresses around the circular openings [5]. Sharan proposed the analytical solutions for stresses and displacements around the circular openings, and obtained different solutions of the displacements by changing the dilation parameters [2]. Chen and Tonon reviewed the works of the aforementioned authors and proposed an improved solution for the rock mass governed by the GHB yield criterion [9]. Meng and Wang[10] presented a novel closed-form solution for circular openings based on GHB yield criterion and shown that the proposed solution was most sensitive to parameter $a_{r}$. Zhang et al. [11] introduced the UST (Unified Strength Theory) into deriving the displacement solution and stress distribution for circular openings. Their solutions have wide application and were proved to be a series of results rather than solutions derived, based on any given single criterion. Lee and Pietruszczak [12] calculated the displacement and stress of circular openings with a successive manner, based on dividing the potential plastic zone into numbers of rings, their numerical approximate solutions agree well with the exact solutions while the width of rings becoming smaller.

It is no doubt, that the aforementioned analyses contribute greatly to the analysis of displacement around circular openings in GHB rock. However, as shown in Fig. 1, the phenomenon of slip lines is easily observed in numerical simulation, similar material simulation and the practical rock engineering. Therefore, both the rock and the displacement field in the plastic region are discontinuous [15].

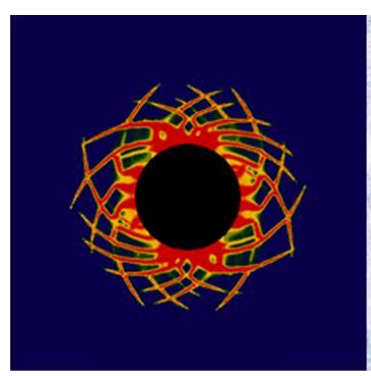

(a)

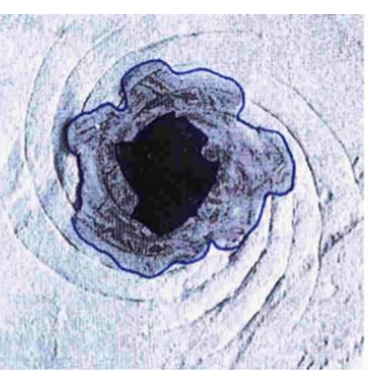

(b)

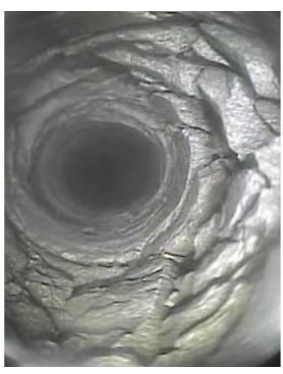

(c)

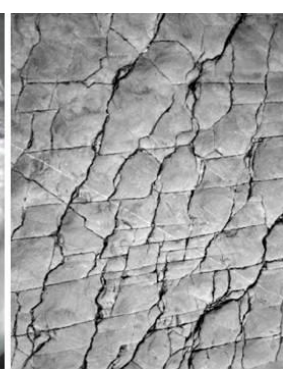

(d)

Fig. 1. (a) Slip lines around circular opening (numerical simulation [13]); (b) Slip lines around circular opening (similar material simulation[14]); (c) Slip lines around the borehole; (d) Slip lines in surrounding rock. 
The deformation in plastic region mainly consists of two parts, one is the elastic deformation of the elements (resulting from scale decease, plasticity limit of the smaller elements is higher than that of the macro rock mass), and another is the irreversible deformation, caused by slippage. Compared with slippage, deformation of the elements is insignificant and can be neglected [15], which means, the deformation of the plastic region is mainly the result of slippage. Slippage-induced deformation is widely observed, however, study towards displacement solution induced by this mechanism is rare.

This paper deals with an analytical solution of the radial displacement around the circular openings in elastic-brittle-plastic rock. The rock is assumed to obey the GHB yield criterion. Different from the aforementioned solutions, the deformation of the plastic region after failure, in this study, is mainly attributed to the slippage rather than the continuous deformation, such as rock dilatation. The relationship between the slippages and the displacement is presented based on the study of Revuzhenko and Shemyakin [16]. Slip lines around the circular openings are simplified as logarithmic spirals. With several assumptions, relations between slippage and rock failure are described based on the elastic-brittle-plastic model. An analytical solution of the radial displacement, which has direct relations with slippage is derived. At the end, displacements around the circular openings in different cases are computed with the derived solution and compared with those in published sources.

\section{DERIVATION OF DISPLACEMENT AROUND CIRCULAR OPENINGS, INDUCED BY SLIPPAGE}

There are localized shear and slip lines, which may divide the plastic rock into large numbers of elements. The elements can slip along the slip lines and the displacement field in the plastic region is discontinuous. To calculate the displacement induced by slippages, the prerequisite is to describe the discontinuities with continuous method [16].

Based on the study of Revuzhenko and Shemyakin [16], with several restraints, the discontinuous displacement field can be described by the combinations of two smooth functions, $\boldsymbol{u}(\boldsymbol{r})$ and $\mathbf{A}(\boldsymbol{r})$. Of which, $\boldsymbol{u}(\boldsymbol{r})$ denotes the averaged original displacement field, and $\mathbf{A}(\boldsymbol{r})$ characterizes the breaks of the original displacement field. $\boldsymbol{e}_{1}$ and $\boldsymbol{e}_{2}$ are orthogonal basis; $\boldsymbol{r}=x_{1} \boldsymbol{e}_{1}+x_{2} \boldsymbol{e}_{2}$ is the vector variable and $\boldsymbol{U}=U_{1} \boldsymbol{e}_{1}+U_{2} \boldsymbol{e}_{2}$ denotes the vector function in plane problem. $\boldsymbol{U}$ is assumed to be smooth enough within the elements and in center of element $\boldsymbol{r}_{i}$, it satisfies $\boldsymbol{u}\left(\boldsymbol{r}_{i}\right)=\boldsymbol{U}\left(\boldsymbol{r}_{i}\right) . \mathbf{A}$ is a second-order tensor with four smooth components [16]

$$
A_{k m}=\frac{\partial u_{k}}{\partial x_{m}}-\frac{\partial U_{k}}{\partial x_{m}} \quad(k, m=1,2) .
$$


The relation between the shear stresses and the slippages on the slip lines is [16]:

$$
\left\{\begin{array}{l}
\frac{\cos 2 \theta}{2}\left(\frac{\partial u_{1}}{\partial x_{1}}-\frac{\partial u_{2}}{\partial x_{2}}\right)+\frac{\sin 2 \theta}{2}\left(\frac{\partial u_{1}}{\partial x_{2}}+\frac{\partial u_{2}}{\partial x_{1}}\right)-\Omega=\frac{\gamma_{1}}{l_{1}}+\frac{1}{4 \mu}\left(\tau_{1}+\tau_{2}\right) \\
\frac{\cos 2 \theta}{2}\left(\frac{\partial u_{1}}{\partial x_{1}}-\frac{\partial u_{2}}{\partial x_{2}}\right)+\frac{\sin 2 \theta}{2}\left(\frac{\partial u_{1}}{\partial x_{2}}+\frac{\partial u_{2}}{\partial x_{1}}\right)+\Omega=\frac{\gamma_{2}}{l_{2}}+\frac{1}{4 \mu}\left(\tau_{1}+\tau_{2}\right)
\end{array}\right.
$$

where

$$
\Omega=\frac{1}{2}\left(A_{12}-A_{21}\right)=\frac{1}{2}\left(\frac{\partial U_{2}}{\partial x_{1}}-\frac{\partial U_{1}}{\partial x_{2}}\right)-\frac{1}{2}\left(\frac{\partial u_{2}}{\partial x_{1}}-\frac{\partial u_{1}}{\partial x_{2}}\right)
$$

represents the difference of curls between the original and the averaged deformation fields.

Let's introduce $\omega$, which is defined as

$$
\omega=\Omega+\frac{1}{2}\left(\frac{\partial u_{2}}{\partial x_{1}}-\frac{\partial u_{1}}{\partial x_{2}}\right)=\frac{1}{2}\left(\frac{\partial U_{2}}{\partial x_{1}}-\frac{\partial U_{1}}{\partial x_{2}}\right) .
$$

As shown in Fig. 2, $\mu$ is shear modulus; $x_{1}$ and $x_{2}$ are basis of the Cartesian coordinates; $u_{1}$ and $u_{2}$ are components of averaged displacement field in directions of $x_{1}$ and $x_{2}$; since slip lines in plastic region around the circular openings are curves, therefore, in order to describe them conveniently, the curvilinear coordinates, $\lambda_{1}$ and $\lambda_{2}$ are adopted. In fact, $\lambda_{1}$ and $\lambda_{2}$ are basis of the curvilinear coordinates, they also represent two orthogonal slip lines; $\theta$ is the included angle between the principal stress and the $o x_{1}$ axis. $l_{1}=f_{1} a_{1}$ and $l_{2}=f_{2} a_{2}$ are the lengths of arcs along the slip lines $\lambda_{1}$ and $\lambda_{2} ; f_{1}$ and $f_{2}$ are distribution functions of slip lines $\lambda_{1}$ and $\lambda_{2}$; $a_{1}=\partial l_{1} / \partial \lambda_{1}$ and $a_{2}=\partial l_{2} / \partial \lambda_{2}$ are the Lame Constants between the curvilinear coordinates and the Cartesian coordinates [17]; $\tau_{1}$ and $\tau_{2}$ are shear stresses on slip lines $\lambda_{1}$ and $\lambda_{2} ; \gamma_{1}$ and $\gamma_{2}$ are slippages of elements along the slip lines $\lambda_{1}$ and $\lambda_{2}$.

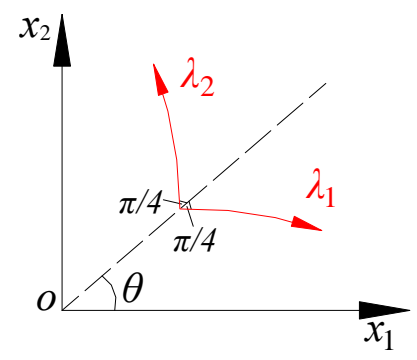

Fig. 2. Curvilinear coordinates in Cartesian coordinates. 


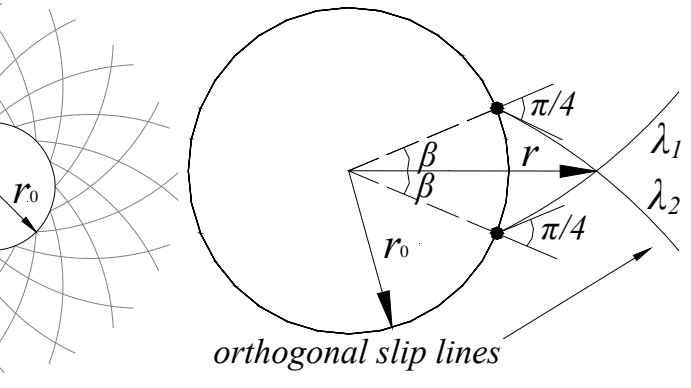

(a)

(b)

Fig. 3. (a) Mathematical model of slip lines; (b) Two of the slip lines around the circular opening [15].

As shown in Fig. 3 (a), ignoring the effects of rock heterogeneity, slip lines in plastic rock, subjected to hydrostatic stress are assumed to be axisymmetric and can be described by the logarithmic spirals in polar coordinates [15]:

$$
\left\{\begin{array}{l}
\lambda_{1}=\frac{1}{\sqrt{2}}\left(\ln \frac{r}{r_{0}}-\beta\right) \\
\lambda_{2}=\frac{1}{\sqrt{2}}\left(\ln \frac{r}{r_{0}}+\beta\right)
\end{array}\right.
$$

As shown in Fig. 3 (b), $\lambda_{1}$ and $\lambda_{2}$ denote two slip lines, $r_{0}$ is the opening radius; the relation between slip line and $\beta$ is shown in the above figure. The Lame Constants between the polar coordinates and the orthogonal curvilinear coordinates are $a_{1}=$ $a_{2}=r$ [17]. Considering the slip lines, the slippages and the displacements are axisymmetric, then $f_{1}=f_{2}=1, l_{1}=l_{2}=r, \tau_{1}=\tau_{2}, \gamma_{1}=\gamma_{2}$ [17]. Besides, in plain problem, based on elasticity theory [18]

$$
\left\{\begin{array}{l}
u_{1}=u_{r} \cos \theta-u_{\theta} \sin \theta \\
u_{2}=u_{r} \sin \theta+u_{\theta} \cos \theta
\end{array} .\right.
$$

Therefore, Eq. (2) can be rewritten, as

$$
\left\{\begin{array}{l}
\frac{\partial}{\partial r}\left(u_{r}+u_{\theta}\right)-\frac{1}{r} \frac{\partial}{\partial \theta}\left(u_{r}+u_{\theta}\right)-\frac{u_{r}-u_{\theta}}{r}-2 \omega=\frac{1}{2 \mu}\left(\sigma_{r}-\sigma_{\theta}\right)+\frac{2}{r} \delta\left(\frac{\sigma_{\theta}-\sigma_{r}}{2}\right) \\
\frac{\partial}{\partial r}\left(u_{r}-u_{\theta}\right)+\frac{1}{r} \frac{\partial}{\partial \theta}\left(u_{r}-u_{\theta}\right)-\frac{u_{r}+u_{\theta}}{r}+2 \omega=\frac{1}{2 \mu}\left(\sigma_{r}-\sigma_{\theta}\right)+\frac{2}{r} \delta\left(\frac{\sigma_{\theta}-\sigma_{r}}{2}\right)
\end{array} .\right.
$$


Eq. (7) can be further simplified, as

$$
\frac{\partial u_{r}}{\partial r}-\frac{u_{r}}{r}=\frac{1}{2 \mu}\left(\sigma_{r}-\sigma_{\theta}\right)+\frac{2 \delta(\tau)}{r},
$$

where $\sigma_{r}$ (or $u_{r}$ ) and $\sigma_{\theta}\left(\right.$ or $u_{\theta}$ ) are the radial and circumferential stresses (or displacements) components, respectively; $\delta($ ) represents an operation; Under the axisymmetric plain condition, we also have the following relations in surrounding rock [18]

$$
\left\{\begin{array}{l}
\frac{\partial \sigma_{r}}{\partial r}+\frac{\sigma_{r}-\sigma_{\theta}}{r}=0 \\
\frac{\partial u_{r}}{\partial r}+\frac{u_{r}}{r}=\frac{1-2 \nu}{2 \mu}\left(\sigma_{r}+\sigma_{\theta}\right)
\end{array}\right.
$$

Therefore, based on Eqs (8) and (9), the dimensionless radial displacement $u_{r} / r$ can be expressed, as

$$
\left\{\begin{array}{l}
\frac{u_{r}}{r}=\frac{1-2 \nu}{\mu} \int\left(\frac{B_{2}}{r^{3}}-\frac{\tau}{r}\right) d r+B_{1} \\
\frac{B_{2}}{r^{3}}=\frac{2 \mu}{1-2 \nu} \frac{\delta(\tau)}{r^{2}}+\frac{2 \tau(1-v)}{1-2 \nu} \frac{1}{r}
\end{array},\right.
$$

where $B_{1}$ and $B_{2}$ are integration constants that depend on the boundary conditions, $v$ is the Poisson's ratio, $\tau=\left(\sigma_{\theta}-\sigma_{r}\right) / 2$ is shear stress.

\section{PROPERTIES OF ROCK MASS AND SIMPLIFIED DEFORMATION PROCESS AROUND CIRCULAR OPENINGS}

As shown in the following Fig. 4, the rock mass is assumed to be homogeneous, isotropic, infinitely large and subjected to a hydrostatic in situ stress $\sigma_{0}$. The initial

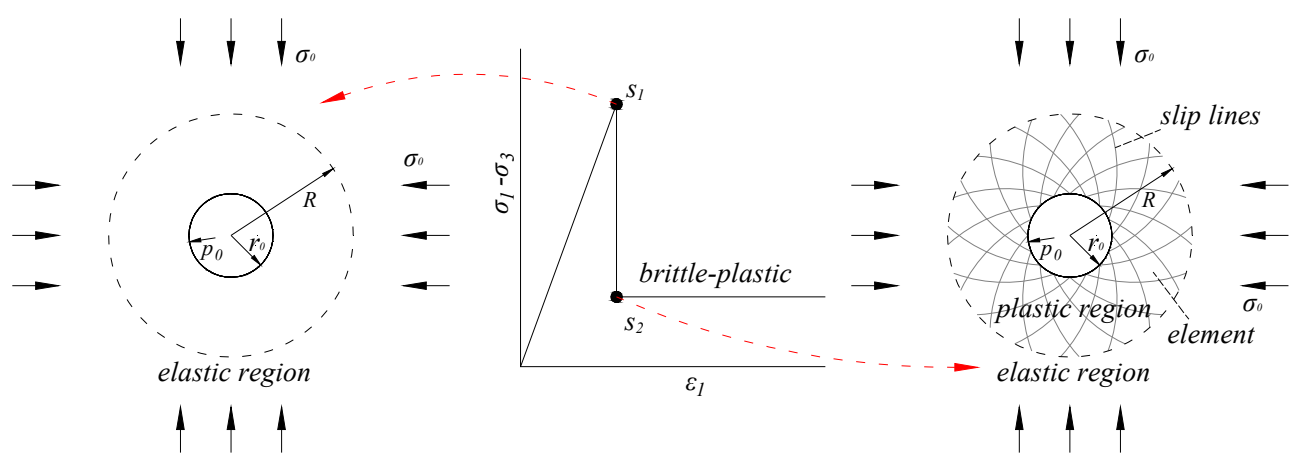

Fig. 4. Slip lines around circular opening and the material behaviour. 
internal pressure acting on the opening surface is $p_{0}$, the rock mass is in elastic state before excavation. The internal pressure is gradually decreased after excavation and the radial displacement will occur. The plastic zone will appear when the elastic limit of the surrounding rock is reached [15]. Based on the elastic-brittle-plastic model, after yielding, the strength of the rock will suddenly drop from the peak value to the residual value, and at the same time, the slip lines and the slippages will develop rapidly in the plastic region.

Figure 4 is description of the simplified deformation process around the circular opening. The total displacement on the opening surface is assumed to be caused by two parts, of which, one is the maximum continuous deformation corresponding to point $s_{1}$. In this case, the shear stresses in the range of $r_{0} \leq r \leq R$ and the displacement on the opening surface both have reached their peak values within the rock's elastic limit; another is the slippages occurred from points $s_{1}$ to $s_{2}$. The rock strength drops suddenly from peak value to residual value from $s_{1}$ to $s_{2}$, and in order to simplify the analyses, both the development of slip lines and the slippages of elements are assumed to be finished at point $s_{2}$.

\section{ANALYSIS OF THE PLASTIC ZONE AROUND CIRCULAR OPENINGS}

The rock mass is assumed to be governed by the GHB yield criterion, which is given by [5]

$$
\sigma_{1}=\sigma_{3}+\sigma_{c i}\left(m_{b} \frac{\sigma_{3}}{\sigma_{c i}}+s\right)^{a},
$$

where

$$
\begin{aligned}
& m_{b}=m_{i} \exp \left(\frac{G S I-100}{28-14 D}\right) \\
& s=\exp \left(\frac{G S I-100}{9-3 D}\right) \\
& a=\frac{1}{2}+\frac{1}{6}\left[\exp \left(\frac{-G S I}{15}\right)-\exp \left(\frac{-20}{3}\right)\right]
\end{aligned}
$$

$\sigma_{c i}$ is the uniaxial compressive strength; $m_{i}$ is the material constant for the intact rock; $G S I$ and $D$ are the Geological Strength Index and disturbance factor of the rock mass, respectively. The ranges of $G S I$ and $D$ are normally as $10 \leq G S I \leq 100$ ( $G S I=10$ corresponds to an extremely poor quality of rock mass) and $0 \leq D \leq 1$ ( $D=1$ corresponds to a highly disturbed rock mass), respectively.

For it is an axisymmetric plane problem, the radial and circumferential stresses in the plastic region are the minor and major principal stresses, respectively, i.e. $\sigma_{r}=$ 
$\sigma_{3}, \sigma_{\theta}=\sigma_{1}$, therefore, Eq. (11) can be rewritten, as:

$$
\sigma_{\theta}=\sigma_{r}+\sigma_{c i}\left(m_{b} \frac{\sigma_{r}}{\sigma_{c i}}+s\right)^{a} .
$$

Based on the stress condition in the elastic zone at the elastic-plastic interface, $r=R$, the radial stress $\sigma_{R}$ at the elastic-plastic interface can be obtained by solving the following equation [9]:

$$
2\left(\sigma_{0}-\sigma_{R}\right)=\sigma_{c i}\left(m_{b} \frac{\sigma_{R}}{\sigma_{c i}}+s\right)^{a} .
$$

For that $a \neq 0.5$, therefore, Eq. (14) must be solved numerically [19]. According to $[7,8]$, the explicit approximate closed-form solution for the radial stress at the elastic-plastic interface is given by

$$
\sigma_{R}=\left(\sigma_{0}-C_{1} \sigma_{c i}\right)+\frac{2 C_{1} \sigma_{c i}-\sigma_{c i} C_{2}^{a}}{2+a m_{b} C_{2}^{a-1}},
$$

where

$$
\begin{aligned}
C_{1} & =\frac{1}{2} \sqrt{\left(\frac{m_{b}}{4}\right)^{2}+m_{b} \frac{\sigma_{0}}{\sigma_{c i}}+s}-\frac{m_{b}}{8}, \\
C_{2} & =m_{b} \frac{\sigma_{0}-C_{1} \sigma_{c i}}{\sigma_{c i}}+s .
\end{aligned}
$$

After failure, the stresses in the plastic zone are governed by the following form of Eq. (11), which is given by

$$
\sigma_{\theta}=\sigma_{r}+\sigma_{c i r}\left(m_{b r} \frac{\sigma_{r}}{\sigma_{c i r}}+s_{r}\right)^{a_{r}} .
$$

By substituting Eq. (13) into the first equation of Eq. (9), the following equation is obtained:

$$
\frac{\partial \sigma_{r}}{\partial r}-\frac{\sigma_{c i}}{r}\left(m_{b} \frac{\sigma_{r}}{\sigma_{c i}}+s\right)^{a}=0 .
$$

By considering the boundary condition

$$
\sigma_{r}=\left\{\begin{array}{ll}
\sigma_{0}, & r \rightarrow \infty \\
p_{0}, & r=r_{0}
\end{array} .\right.
$$

The solutions for the radial stresses, corresponding to the peak and residual strengths of the rock mass, can be expressed, as: 
- for peak strength

$$
\sigma_{r 1}=\frac{\left[\left(C_{40} p_{0}+s_{1}\right)^{C_{30}}+C_{30} m_{b} \ln \left(r / r_{0}\right)\right]^{1 / C_{30}}-s_{1}}{C_{40}} ;
$$

- for residual strength

$$
\sigma_{r 2}=\frac{\left[\left(C_{4} p_{0}+s_{2}\right)^{C_{3}}+C_{3} m_{b r} \ln \left(r / r_{0}\right)\right]^{1 / C_{3}}-s_{2}}{C_{4}},
$$

where

$$
C_{30}=1-a, \quad C_{3}=1-a_{r}, \quad C_{40}=\frac{m_{b}}{\sigma_{c i}}, \quad C_{4}=\frac{m_{b r}}{\sigma_{c i r}}
$$

and $\sigma_{c i r}, m_{b r}, s_{2}, a_{r}$ are the residual values of $\sigma_{c i}, m_{b}, s_{1}, a$. By substituting Eq. (20) into Eq. (13), and Eq. (21) into Eq. (17), the circumferential stresses corresponding to the peak and the residual strength of the rock mass can be obtained.

The expression for the radius of the elastic-plastic interface $R$ can be derived by solving the equation obtained by substituting $r=R$ and $\sigma_{r}=\sigma_{R}$ into Eq.(20), the result is

$$
R=r_{0} \exp \left(\frac{C_{5}-C_{6}}{C_{3} m_{b r}}\right)
$$

where

$$
C_{5}=\left(C_{4} \sigma_{R}+s_{2}\right)^{C_{3}}, \quad C_{6}=\left(C_{4} p_{0}+s_{2}\right)^{C_{3}} .
$$

The radial and circumferential stresses in the elastic region are given by the Lame solution and can be expressed, as

$$
\left\{\begin{array}{l}
\sigma_{r e}=\sigma_{0}-\left(\frac{R}{r}\right)^{2}\left(\sigma_{0}-\sigma_{R}\right) \\
\sigma_{\theta e}=\sigma_{0}+\left(\frac{R}{r}\right)^{2}\left(\sigma_{0}-\sigma_{R}\right)
\end{array}\right.
$$

where $\sigma_{r e}$ and $\sigma_{\theta e}$ are the radial and circumferential stress components in the elastic region, respectively, according to Eqs (13) and (17) and $\tau=\left(\sigma_{\theta}-\sigma_{r}\right) / 2$, the shear stresses $\tau_{p 1}, \tau_{p 2}$ in ranges of $r_{0} \leq r \leq R$, and $\tau_{e}$ in ranges of $r>R$ are given as:

- for peak strength in range of $r_{0} \leq r \leq R$

$$
\tau_{p 1}=\frac{1}{2} \sigma_{c i}\left(m_{b} \frac{\sigma_{r 0}}{\sigma_{c i}}+s_{1}\right)^{a} ;
$$


- for residual strength in range of $r_{0} \leq r \leq R$

$$
\tau_{p 2}=\frac{1}{2} \sigma_{c i r}\left(m_{b r} \frac{\sigma_{r 1}}{\sigma_{c i r}}+s_{2}\right)^{a_{r}} ;
$$

- shear stress in range of $r>R$

$$
\tau_{e}=\left(\frac{R}{r}\right)^{2}\left(\sigma_{0}-\sigma_{R}\right) .
$$

According to Eq. (10) and Eqs (26) - (28), when there is plastic region around circular openings, the new analytical solution for the dimensionless radial displacement on the opening surface can be expressed as

$$
\frac{u_{0}}{r_{0}}=2 \frac{\delta(\tau)}{l} \int_{r_{0}}^{R} \frac{r_{0}}{r^{2}} d r+\frac{1}{\mu} \int_{r_{0}}^{R} \frac{\tau_{p 1}}{r} d r+\frac{1}{\mu} \int_{R}^{r} \frac{\tau_{e}}{r} d r .
$$

If the rock around the circular opening is elastic, there is no slip line, i.e., $\delta(\tau) / l=$ 0 , in this case, the dimensionless radial displacement around the circular opening can be expressed as

$$
\frac{u_{0}}{r_{0}}=\frac{1}{\mu} \int_{r_{0}}^{r} \frac{\tau_{e}}{r} d r .
$$

In this case, the shear stress in the range of $r \geq r_{0}$ can be expressed as $\tau_{e}=$ $\left(\sigma_{0}-p_{0}\right) r_{0}^{2} / r^{2}$, let $r \rightarrow \infty$, then Eq. (30) can be rewritten as

$$
\frac{u_{0}}{r_{0}}=\frac{1}{\mu} \int_{r_{0}}^{r} \frac{\tau_{e}}{r} d r=\frac{\sigma_{0}-p_{0}}{2 \mu} .
$$

Similarly, if there is plastic region around the circular opening, the dimensionless radial displacement at the elastic-plastic interface can be expressed as

$$
\frac{u_{R}}{R}=\frac{1}{\mu} \int_{R}^{r} \frac{\tau_{e}}{r} d r=\frac{\sigma_{0}-\sigma_{R}}{2 \mu} .
$$

It is obvious that Eqs (31) and (32) are the same with that obtained by using the elastic analysis of the infinite rock mass. In Eq (29), $u_{0} / r_{0}$ is the dimensionless radial displacement on the opening surface; the first term on the right-hand side of Eq. (29) represents the dimensionless radial displacement, caused by the slippage. $\delta(\tau) / l$ is ratio of the slippage to the length of the element, its value approximates the axial strain of standard cylindrical specimen in the situation, when the localized shear is completed. The second term on the right-hand depicts the dimensionless radial displacement, corresponding to the situation that the shear stresses in the range of $r_{0} \leq r \leq R$ reach their maximum values within the elastic limit. The last term characterizes the dimensionless radial displacement, caused by the elastic strain in the range of $r>R$. 


\section{EXAMPLES AND RESULTS}

As shown in Table 1, in order to show the results obtained by using the new analytical solution and the effect of the slippage parameter on the displacement, five different examples were considered, the basic mechanical properties of the rock masses are mainly taken from published sources. Examples 1-2 correspond to very good and averaged qualities of rock masses, Example 3 corresponds to very poor quality of rock mass. For all examples, it was assumed that $D=0$. The values of GHB constants are computed by using Eq. (12) and are shown in Table 2, the residual values of the constants in Examples 1-2 are computed, based on the assumptions that $D_{r}=0.5, v_{r}=v, \sigma_{c i r}=\sigma_{c i}$ and $G S I_{r}=0.5 G S I$. The residual values with subscript " $r$ " are shown in Table 2. Considering that the extent disturbed by the excavation is limited, thus, we only analyze the deformation within the range $r_{0} \leq r \leq 20 r_{0}$.

Table 1. Properties of rock mass and data for underground openings used for test example cases

\begin{tabular}{cclccccccc}
\hline \hline Example & Ref. & $\begin{array}{l}\text { Quality of } \\
\text { rock mass }\end{array}$ & $G S I$ & $\begin{array}{c}\sigma_{c i} \\
(\mathrm{Mpa})\end{array}$ & $\begin{array}{c}\sigma_{c i r} \\
(\mathrm{Mpa})\end{array}$ & $\begin{array}{c}E \\
(\mathrm{Gpa})\end{array}$ & $\begin{array}{c}E_{r} \\
(\mathrm{Gpa})\end{array}$ & $v$ & $m_{i}$ \\
\hline 1 & {$[20]$} & Very good & 75 & 150 & 150 & 42 & 10 & 0.2 & 25 \\
2 & {$[20]$} & Average & 50 & 80 & 80 & 9 & 5 & 0.25 & 12 \\
3 & {$[19]$} & Very poor & - & 30 & 25 & 5.7 & 5.7 & 0.3 & - \\
\hline
\end{tabular}

Table 2. Hoek-Brown constants obtained from Table 1

\begin{tabular}{ccccccc}
\hline \hline Example & $a$ & $m_{b}$ & $s_{1}$ & $a_{r}$ & $m_{b r}$ & $s_{2}$ \\
\hline 1 & 0.5 & 10.2 & 0.062 & 0.51 & 1.27 & 0.0002 \\
2 & 0.51 & 2.01 & 0.0039 & 0.53 & 0.34 & 0 \\
3 & 0.55 & 1.7 & 0.0039 & 0.6 & 0.85 & 0.0019 \\
\hline
\end{tabular}

As shown in the following Tables $3-5$, the rock behaviour is brittle-plastic, the slippage parameter, the extent of the plastic region and the radial displacement are presented in terms of dimensionless forms $\delta(\tau) / l, R / r_{0}$ and $u_{0} / r_{0}$, respectively. The results in Table 3 (or 4 ) are obtained by substituting the parameters in Example 1 (or 2) into the above Eqs (23) and (29). Table 5 is obtained by substituting the parameters in Example 3 into Eqs (23) and (29). Tables 3-5 show that the results obtained by using our method agree well with the exact solutions in published sources.

Table 3 shows that with the plastic region kept invariantly, the displacement $u_{0} / r_{0}$ on the opening surface is increased as the slippage parameter $\delta(\tau) / l$ increase. For ex- 
Table 3. Comparison of results for a very good quality rock mass (Example 1 [15]). Data in parenthesis are exact solutions from reference [15]

\begin{tabular}{clcccccc}
\hline \hline No. & $\begin{array}{l}\text { Plastic } \\
\text { behaviour }\end{array}$ & $\begin{array}{c}\sigma_{0} \\
(\mathrm{Mpa})\end{array}$ & $\begin{array}{c}p_{0} \\
(\mathrm{Mpa})\end{array}$ & $R / r_{0}$ & $\delta(\tau) / l$ & $\begin{array}{c}u_{0} / r_{0} \\
(\%)\end{array}$ & $\begin{array}{c}\text { Error } \\
(\%)\end{array}$ \\
\hline $\mathbf{1}$ & brittle plastic & 37.5 & 0 & 1.23 & 0.0026 & $0.27(0.27)$ & 0 \\
2 & brittle plastic & 37.5 & 0 & 1.23 & 0.0036 & $0.31(0.31)$ & 0 \\
3 & brittle plastic & 37.5 & 0 & $\mathbf{1 . 2 3}$ & $\mathbf{0 . 0 1 2 8}$ & $0.66(0.66)$ & 0 \\
$\mathbf{4}$ & brittle plastic & 75 & 0 & $\mathbf{1 . 5 8}$ & $\mathbf{0 . 0 0 8 6}$ & $\mathbf{1 . 0 9}(1.09)$ & 0 \\
$\mathbf{5}$ & brittle plastic & 75 & 0 & $\mathbf{1 . 5 8}$ & $\mathbf{0 . 0 1 2 8}$ & $\mathbf{1 . 4 0}(1.40)$ & 0 \\
$\mathbf{6}$ & brittle plastic & 75 & 0 & $\mathbf{1 . 5 8}$ & $\mathbf{0 . 0 6 2 0}$ & $\mathbf{5 . 0 1}(5.01)$ & 0 \\
$\mathbf{7}$ & brittle plastic & 150 & 0 & $\mathbf{2 . 3 4}$ & $\mathbf{0 . 0 3 5 7}$ & $\mathbf{5 . 3 0}(5.30)$ & 0 \\
$\mathbf{8}$ & brittle plastic & 150 & 0 & $\mathbf{2 . 3 4}$ & $\mathbf{0 . 0 5 8 8}$ & $\mathbf{7 . 9 4}(7.94)$ & 0 \\
9 & brittle plastic & 150 & 2 & 1.93 & 0.0371 & $4.49(4.49)$ & 0 \\
$\mathbf{1 0}$ & brittle plastic & 150 & 0 & $\mathbf{2 . 3 4}$ & $\mathbf{0 . 6 0 8 3}$ & $\mathbf{7 0 . 9}(70.9)$ & 0 \\
11 & brittle plastic & 150 & 12 & 1.42 & 0.0621 & $4.28(4.28)$ & 0 \\
\hline
\end{tabular}

Table 4. Comparison of results for a very good quality rock mass (Example 2 [15]). Data in parenthesis are exact solutions from reference [15]

\begin{tabular}{clcccccc}
\hline \hline No. & $\begin{array}{l}\text { Plastic } \\
\text { behaviour }\end{array}$ & $\begin{array}{c}\sigma_{0} \\
(\mathrm{Mpa})\end{array}$ & $\begin{array}{c}p_{0} \\
(\mathrm{Mpa})\end{array}$ & $R / r_{0}$ & $\delta(\tau) / l$ & $\begin{array}{c}u_{0} / r_{0} \\
(\%)\end{array}$ & $\begin{array}{c}\text { Error } \\
(\%)\end{array}$ \\
\hline $\mathbf{1 2}$ & brittle plastic & 20 & 0 & $\mathbf{2 . 8 9}$ & $\mathbf{0 . 0 1 6 0}$ & $\mathbf{2 . 9 6}(2.96)$ & 0 \\
$\mathbf{1 3}$ & brittle plastic & 20 & 0 & $\mathbf{2 . 8 9}$ & $\mathbf{0 . 0 1 9 6}$ & $\mathbf{3 . 4 4}(3.44)$ & 0 \\
$\mathbf{1 4}$ & brittle plastic & 20 & 0 & $\mathbf{2 . 8 9}$ & $\mathbf{0 . 0 5 5 1}$ & $\mathbf{8 . 0 8}(8.08)$ & 0 \\
15 & brittle plastic & 20 & 1 & 1.78 & 0.0140 & $1.73(1.73)$ & 0 \\
$\mathbf{1 6}$ & brittle plastic & 40 & 0 & 5.65 & 0.1106 & $20.3(20.3)$ & 0 \\
17 & brittle plastic & 40 & 3 & 2.53 & 0.0215 & $3.70(3.70)$ & 0 \\
18 & brittle plastic & 40 & 0 & 5.65 & 0.1440 & $25.8(25.8)$ & 0 \\
19 & brittle plastic & 40 & 3 & 2.53 & 0.0256 & $4.19(4.19)$ & 0 \\
20 & brittle plastic & 40 & 0 & 5.65 & 0.6190 & $104(104)$ & 0 \\
21 & brittle plastic & 40 & 5 & 2.03 & 0.0340 & $4.35(4.34)$ & -0.18 \\
$\mathbf{2 2}$ & brittle plastic & 80 & 0 & $\mathbf{1 5 . 1 4}$ & $\mathbf{1 . 2 7 1 1}$ & $\mathbf{2 4 2}(242)$ & 0 \\
23 & brittle plastic & 80 & 18 & 2.33 & 0.0298 & $5.00(4.99)$ & -0.11 \\
$\mathbf{2 4}$ & brittle plastic & 80 & 0 & $\mathbf{1 5 . 1 4}$ & $\mathbf{1 . 8 6 0 0}$ & $\mathbf{3 5 2}(352)$ & 0 \\
25 & brittle plastic & 80 & 20 & 2.12 & 0.0281 & $4.44(4.44)$ & 0 \\
$\mathbf{2 6}$ & brittle plastic & 80 & 0 & $\mathbf{1 5 . 1 4}$ & $\mathbf{1 7 . 3 7 5 0}$ & $\mathbf{3 2 5 0}(3250)$ & 0 \\
27 & brittle plastic & 80 & 24 & 1.78 & 0.0371 & $4.49(4.49)$ & 0 \\
\hline
\end{tabular}


Table 5. Comparison of results for a very good quality rock mass (Example 3 [19]). Data in parenthesis are exact solutions from reference [19]

\begin{tabular}{clcccccc}
\hline \hline No. & $\begin{array}{l}\text { Plastic } \\
\text { behaviour }\end{array}$ & $\begin{array}{c}\sigma_{0} \\
(\mathrm{Mpa})\end{array}$ & $\begin{array}{c}p_{0} \\
(\mathrm{Mpa})\end{array}$ & $R / r_{0}$ & $\delta(\tau) / l$ & $\begin{array}{c}u_{0} / r_{0} \\
(\%)\end{array}$ & $\begin{array}{c}\text { Error } \\
(\%)\end{array}$ \\
\hline 28 & brittle plastic & 8 & 0 & 2.44 & 0.0051 & $0.94(0.94)$ & 0 \\
29 & brittle plastic & 8 & 0 & 2.44 & 0.0147 & $2.07(2.08)$ & 0.28 \\
30 & brittle plastic & 15 & 0 & 3.90 & 0.0225 & $4.06(4.06)$ & 0 \\
31 & brittle plastic & 15 & 3 & 1.51 & 0.0025 & $0.52(0.52)$ & 0 \\
32 & brittle plastic & 15 & 0 & 3.90 & 0.0867 & $13.6(13.6)$ & 0 \\
33 & brittle plastic & 15 & 1 & 2.28 & 0.0192 & $2.65(2.65)$ & 0 \\
34 & brittle plastic & 30 & 0 & 7.84 & 0.1518 & $28(28)$ & 0 \\
35 & brittle plastic & 30 & 3 & 3.03 & 0.0220 & $3.92(3.92)$ & 0 \\
36 & brittle plastic & 30 & 0 & 7.84 & 0.9943 & $175(175)$ & 0 \\
37 & brittle plastic & 30 & 5 & 2.33 & 0.0323 & $4.51(4.51)$ & 0 \\
\hline
\end{tabular}

Nnote: exact solutions refer to solutions, obtained by exact integration method with mathematical software.

ample, according to Nos. 1-3, with the slippage parameter increasing from 0.0026 to 0.0128 , the displacement on the opening surface will increase from $0.27 \%$ to $0.66 \%$, i.e., with the other conditions kept unchanged, the displacement increases obviously with the increase of the slippage parameter. This conclusion can be also obtained from Nos. 4-6, Nos. 7, 8, 10, Nos. 12-14, Nos. 16, 18, 20, Nos. 22, 24, 26.

Tables $4-5$ show, that although in different rock qualities and under different stress conditions, the displacements on the opening surface obtained by using the new presented method are almost the same with those of the exact solutions.

The above Tables 3-5 also show that, based on the elastic-brittle-plastic model, the analytical solutions presented in this paper can give satisfactory results for the displacements around the circular opening, no matter the quality of the rock mass is very good, averaged or very poor. In addition, compared with the range of the plastic region, the effects of slippage parameter on the displacement is more obvious, i. e., the displacement can have very significant change even the change of the slippage parameter is slight, which means it is reasonable for us to attribute the displacement in plastic region to the slippage.

\section{CONCLUSION}

In this paper, based on the displacement in plastic region after failure is mainly the result of slippage; the relationship between slippage and shear stress is introduced; the relations between slippage and rock failure is established, based on the elastic- 
brittle-plastic model. A new analytical solution of radial displacement around the circular openings is presented, and the following conclusions are drawn:

1. When there is plastic region around the circular openings, the radial displacement on the opening surface can be obtained by using Eq. (29), if the whole surrounding rock is elastic, the radial displacement can be computed by using Eq. (30).

2. Compared with the range of the plastic region, the effect of the slippage on the displacement is more significant. A slight change in slippage parameter can result in obvious change in radial displacement. Therefore, it is reasonable to attribute the main displacement in plastic region to slippage.

\section{ACKNOWLEDGMENTS}

The authors would like to express their sincere gratitude to the financial support by the National Natural Science Foundation of China (Grant No.2013CB036005, $51527810,51679249)$, in addition, their appreciation also goes to the editor and the anonymous reviewers for their comments.

\section{REFERENCES}

[1] Sharan, S. K. Exact and Approximate Solutions for Displacements around Circular Openings in Elastic-brittle-plastic Hoek-Brown Rock. Int. J. Rock Mech. Min. Sci., 42 (2005), 542-549.

[2] Sharan, S. K. Analytical Solutions for Displacements around Circular Openings in a Generalized Hoek-Brown Rock. Int. J. Rock Mech. Min. Sci., 45 (2008), 78-85.

[3] Brown, E. T., J. W. Bray, B. Ladanyi, E. Hoek. Ground Response Curves for Rock Tunnels. ASCE J. Geotech. Eng., 109 (1983), 15-39.

[4] Wang, Y. Ground Response of Circular Tunnel in Poorly Consolidated Rock. ASCE J. Geotech. Eng., 122 (1996), 703-708.

[5] Carranze-Torres, C. Elastoplastic Solution of Tunnel Problems using the Generalized Form of Hoek-Brown Failure Criterion. Int. J. Rock Mech. Min. Sci., 41 (2004), 629-639.

[6] PARK, K. H., Y. J. Kim. Analytical Solution for a Circular Opening in an Elasticbrittle-plastic Rock. Int. J. Rock Mech. Min. Sci., 43 (2006), 616-622.

[7] Sofianos, A. I., P. P. Nomikos. Equivalent Mohr-Coulomb and Generalized HoekBrown Strength Parameters for supported Axisymmetric Tunnels in Plastic or Brittle Rock. Int. J. Rock Mech. Min. Sci., 43 (2006), 683-704.

[8] Sofianos, A. I. Tunneling Mohr-Coulomb Strength Parameters for Rock massed Satisfying the Generalized Hoek-Brown Criterion. Int. J. Rock Mech. Min. Sci., 40 (2003), 435-440. 
[9] Chen, R., F. Tonon. Closed-Form Solutions for a Circular Tunnel in Elstic-brittleplastic Ground with the Original and Generalized Hoek-Brown Failure Criteria. Rock Mech. Rock Eng., 44 (2011), 169-178.

[10] Meng, Q. X., W. Wang. A Novel Closed-form Solution for Circular Openings in Generalized Hoek-Brown Media. Math. Probl. Eng., ID 8708835 (2014), 1-8.

[11] Zhang, C. G., J. H. Zhao, J. W. ZhaO, Q. H. Zhang, X. D. Hu. A New Closedform Solution for Circular Openings modelled by the Unified Strength Theory and Radius-dependent Young's Modulus. Comput. Geotech., 42 (2012), 118-128.

[12] Lee, Y. K., S. Pietruszczak. A New Numerical Procedure for Elasto-plastic Analysis of a Circular Opening Excavated in Strain-softening Rock Mass. Tunn. Undergr. Sp. Tech., 23 (2008), 588-589.

[13] Andre, L. M., E. A. V. JR, J. G. Clemente. Numerical Simulation of Solids Production in Slip-lines Type Breakout Modes using Standard and Cosserat Continua. $J$. Petrol. Sci. Eng., 122 (2014), 134-148.

[14] VAN DEN HoEk, P. J. Prediction of Different Types of Cavity Failure using Bifurcation Theory, 38th Symposium of the American Rock Mechanics Association, Elsworth, Tinucci \& Heasley, 2001, 45-52.

[15] Kachanov, L. M. Foundation of the Theory of Plasticity, Moskow, Nauka, 1969.

[16] Revuznenko, A. F., E. I. Shemyakin. Problem of Plane Strain of Hardening and Softening Plastic Materials. J. Appl. Mech. Tech. Phys., 18 (1977), 406-420.

[17] Huang, K. Z., M. D. Xue, W. M. Lu. Tensor Analysis, Beijing, TsingHua Press, 2003.

[18] Timoshenko, S. P., J. N. Goodner. Theory of Elasticity, Beijing, Higher Education Press, 2013.

[19] Carranze-Torres, C., C. Fairhurst. The Elastic-plastic Response of Underground Excavations in Rock Masses that Satisfy the Hoek-Brown Failure Criteria. Int. J. Rock Mech. Min. Sci., 36 (1999), 777-809.

[20] Hoek, E., E. T. Brown. Practical Estimates of Rock Mass Strength. Int. J. Rock Mech. Min. Sci., 34 (1997), 1165-1186. 